\title{
Arithmetical signatures of the dynamics of the Hénon map
}

\author{
Antônio Endler ${ }^{1, *}$ and Jason A. C. Gallas $1,2,3, \dagger$ \\ ${ }^{1}$ Instituto de Física, Universidade Federal do Rio Grande do Sul, 91501-970 Porto Alegre, Brazil \\ ${ }^{2}$ Institut für Computer Anwendungen, Universität Stuttgart, Pfaffenwaldring 27, D-70569 Stuttgart, Germany \\ ${ }^{3}$ Departamento de Física, Faculdade de Ciências, Universidade de Lisboa, 1749-016 Lisboa, Portugal
}

(Received 10 August 2001; published 6 March 2002)

\begin{abstract}
We report a fourth-degree polynomial that parametrizes analytically all period-4 orbits of the Hénon map and use it to investigate arithmetical signatures of the symbolic coding for this prototypical multidimensional system. A discontinuity in the symbolic dynamics observed by Hansen while following numerically a period6 orbit along a closed loop in parameter space is shown to exist already for period 4. We obtain an analytical expression for the locus of all such discontinuities in parameter space and explain their origin. Our analytical results allow the accurate location of all discontinuities, in contrast with topological methods based on homoclinic tangencies that exist over continuous intervals.
\end{abstract}

DOI: 10.1103/PhysRevE.65.036231

PACS number(s): 05.45. $-\mathrm{a}, 03.65 . \mathrm{Fd}, 45.05 .+\mathrm{x}$

\section{INTRODUCTION}

An outstanding problem in chaotic dynamics is to specify generating partitions for symbolic dynamics in dimensions larger than one [1]. It has been known that the infinite number of unstable periodic orbits embedded in the chaotic invariant set provides sufficient information for estimating the generating partition. However, the extraction of this information is a quite difficult task that is currently the subject of much work [1-12]. Knowledge of the generating partitions is of fundamental importance to understand the evolution of such systems by encoding arbitrary trajectories as an infinite sequence of symbols.

Partitions are "generating" when they do not assign the same symbolic sequence to different orbits. A comparison of several possible generating partitions for the paradigmatic guinea pig, the Hénon map, has been recently reported by Eisele [13] where the difficulties of them are discussed in detail. The main problem is that we only know how to construct generating partitions for the most simple dynamical systems, namely, one-dimensional maps and uniformly hyperbolic systems. Even for two-dimensional dissipative maps there is no systematic way to construct generating partitions, in general.

Various approaches have been introduced to encode a given trajectory in phase space [1-13]. A popular way of obtaining binary partitions is by using the concept of homoclinic tangencies [14], i.e., points on the attractor where the stable and unstable manifolds are parallel. The basic idea is that the binary coding assigned to each periodic orbit remains unchanged when parameters of the dynamical system are varied smoothly. The key aspect of this is the identification of some parameter $p_{h}$ such that the corresponding dynamics is characterized by a complete horseshoe. The encoding of each periodic orbit for any desired parameter $p$ is then obtained by smoothly deforming the orbit from $p_{h}$ to $p$.

From classic works of Cauchy and Puiseux and, specially,

\footnotetext{
*Email address: aendler@if.ufrgs.br

${ }^{\dagger}$ Email address: http://www.if.ufrgs.br/ jgallas
}

Riemann and Weierstrass, we do not expect such smooth variations to work, in general, for arbitrary deformations. Indeed, while studying his box-within-box structuring, Mira [15] observed that for the Hénon map

$$
(x, y) \mapsto\left(a-x^{2}+b y, x\right) .
$$

"There exist a set of boxes such that starting from a box of the $b=0$ axis belonging to this set, and following a correctly selected continuous path in the $(a, b)$ [parameter] plane, it is possible to get to a different box of $b=0$." Later on, Hansen [16] (see also Giovannini and Politi [17]), investigated a specific period-6 orbit of the Hénon map which, when followed along closed paths in parameter space, is transformed into a different orbit, thus signaling to unavoidable ambiguities in the symbolic coding. Such transformation into a different orbit means that somewhere along the deformation path, the symbolic coding of the orbit has to be somehow modified discontinuously in order to yield the final coding.

The discontinuities reported in Refs. $[16,17]$ were found by numerical calculations. Hansen [16] considered the dynamical changes undergone by two of the nine possible period- 6 orbits. As recently found, the set of nine period- 6 orbits has the remarkable property of containing nonlinearly interdependent orbits that display a generic "orbit-withinorbit" stratification $[18,19]$.

The discontinuity in the symbolic coding reported by Hansen [16] was observed while circulating around a period-6 cuspidal structure in parameter space. Hansen stated that period 6 would be the simplest example leading to such discontinuous symbolic change. However, a cuspidal structure is known $[15,20,21]$ to appear already for period 4 . This fact and the very interesting results of Hansen [16] and Giovannini and Politi [17] raise the question whether the simpler and less numerous (only three) period-4 orbits would allow one to gain insight about the difficulties of establishing generating partitions for multidimensional systems.

Apart from the aforementioned interest for practical applications, an additional motivation for investigating the origin of discontinuous changes is provided by a number of questions of concerning the arithmetical nature of periodic 
orbits and their evolution as parameter changes. For instance, it would be very useful for applications to know whether or not the Galois group associated with minimal polynomials defining periodic trajectories are preserved upon changes of parameters. Is there an arithmetical coding capable of labeling different trajectories unambiguously, based possibly on the number-theoretical structure of the orbital points? Can we hope to devise algorithms capable of generating such structuring and labeling? From a mathematical point of view a direct arithmetic labeling would be the most basic that one could possibly use. The answers to these and similar questions $[18,19,21]$ presuppose detailed arithmetical investigations of both orbits (phase space) and parameters (phase diagrams) and this paper may be regarded as a step toward such goals.

Although the familiar topological approach is very useful to describe the relatively tame changes that occur as parameters are varied smoothly, the most interesting physical phenomena appear invariably along curves and wrinkles that cannot be ironed away. The analytical determination of all period-4 wrinkles (nonhyperbolic parameters) was reported recently in Ref. [21]. For particular eigenvalues, such wrinkles define boundaries of stability and multistability domains.

The aim of the present paper is to consider the very rich dynamics along wrinkles and several additional loci that underly period-4 orbits. More precisely, we report an investigation of the changes undergone by all three period- 4 orbits of the Hénon map [Eq. (1)] when the parameters $(a, b)$ are changed adiabatically. We derive an exact analytical expression for the critical parameter locus characterized by orbital discontinuities. In addition, we explain the origin of the orbital discontinuities responsible for ambiguities in the symbolic coding.

In the following section, we show how to obtain explicitly an exact analytical expression parameterizing all possible period-4 orbits of the Hénon map, their solutions and discuss several parameter loci underlying them, pointing out the existence of a cusp in parameter space. In Sec. III, we consider a circulation along a closed triangular parameter path enclosing the cusp, pointing out the existence of three changes in the orbital labeling during the circulation. In Sec. IV, we derive the conditions for the existence of discontinuities, locate precisely where the discontinuity occurs along the triangular path and explain the origin of all such discontinuities. Finally, in Sec. V, we present our conclusions. The Appendix contains a plethora of algebraic details concerning the symmetries of period-4 orbits and identities (automorphisms) implied by them.

\section{PARAMETERIZATION OF ALL PERIOD-FOUR ORBITS}

Any $k$-periodic orbit consists of a set $\left\{x_{j}\right\}, j$ $=1,2, \ldots, k$, of $k$ points that may be used to build a polynomial of degree $k$ whose coefficients, say $\left\{\theta_{\ell}\right\}$, are given by the well-known symmetric functions of the points in $\left\{x_{j}\right\}$.

Now, the equation of motion implies additional relations, constraints, among the $k$ orbital points $\left\{x_{j}\right\}$. These con- straints may be used to express all coefficients $\left\{\theta_{\ell}\right\}$ in terms of any one of them, say $\theta_{m}$. The same constraints may be also used to obtain an additional equation from which all possible values of the chosen $\theta_{m}$ may be determined. In other words, it is always possible to reduce the equations of motion to: (i) a polynomial that parameterizes simultaneously all orbits of any given period in terms of an arbitrarily selected $\theta_{m}$, (ii) a polynomial from which all allowed values of $\theta_{m}$ may be derived. This general procedure is valid for any dynamical system with equations of motion defined by algebraic equations.

As an illustration of the general procedure above, we present now explicit results of a parameterization of all period-4 motions for the Hénon map, Eq. (1). The convenient coefficient $\theta_{m}$ chosen here for this parameterization is the sum $\sigma \equiv x_{1}+x_{2}+x_{3}+x_{4}$ of the orbital points.

Using Eq. (1) to obtain relations interconnecting the orbital points, one finds that all period-4 orbits of the Hénon map have their orbital points defined necessarily by the roots of the polynomial

$$
P(x)=x^{4}-\sigma x^{3}+\theta_{2}(\sigma) x^{2}-\theta_{1}(\sigma) x+\theta_{0}(\sigma),
$$

where

$$
\begin{gathered}
\theta_{2}(\sigma)=\frac{1}{2}[\sigma(\sigma+1-b)-4 a] \\
\theta_{1}(\sigma)=\frac{1}{24}\left[4 \sigma^{3}+12(1-b) \sigma^{2}-40 a \sigma+8(1-b)(1+b)^{2}\right] \\
\theta_{0}(\sigma)=\frac{1}{24}\left\{\sigma^{4}+6(1-b) \sigma^{3}+\left[3(1+b)^{2}-16 a\right] \sigma^{2}\right. \\
-(1-b)\left[12 a-14(1+b)^{2}\right] \sigma+24 a^{2}-24(1+b)^{2} \\
\times(a-b)\}
\end{gathered}
$$

The possible values of $\sigma$ are defined by the roots of the cubic

$$
\sigma^{3}-3 U \sigma-2 V=0
$$

where $U \equiv 4 a / 3-(1+b)^{2}$ and $V \equiv 2(1-b)(1+b)^{2}$ and the discriminant of the cubic is $-2^{2} 3^{3}\left(V^{2}-U^{3}\right)$. Equation (6) shows at once that, for any given pair $(a, b)$ of parameters, the Hénon map contains a total of three period- 4 orbits.

\section{A. The orbital points}

To have a period-4 orbit for the Hénon map means to deal with four numbers $x_{1}, x_{2}, x_{3}, x_{4}$ interconnected by Eq. (1) in the following way:

$$
\left(\begin{array}{l}
x_{1} \\
x_{4}
\end{array}\right) \rightarrow\left(\begin{array}{l}
x_{2} \\
x_{1}
\end{array}\right) \rightarrow\left(\begin{array}{l}
x_{3} \\
x_{2}
\end{array}\right) \rightarrow\left(\begin{array}{l}
x_{4} \\
x_{3}
\end{array}\right) \rightarrow\left(\begin{array}{l}
x_{1} \\
x_{4}
\end{array}\right),
$$

a very specific ordering that repeats forever as time evolves.

In the Hamiltonian case, when $b= \pm 1$, the orbital points $x_{i}$ may be easily obtained since $V=0$ along both lines $b=$ \pm 1 . Then, Eq. (6) simplifies considerably, 

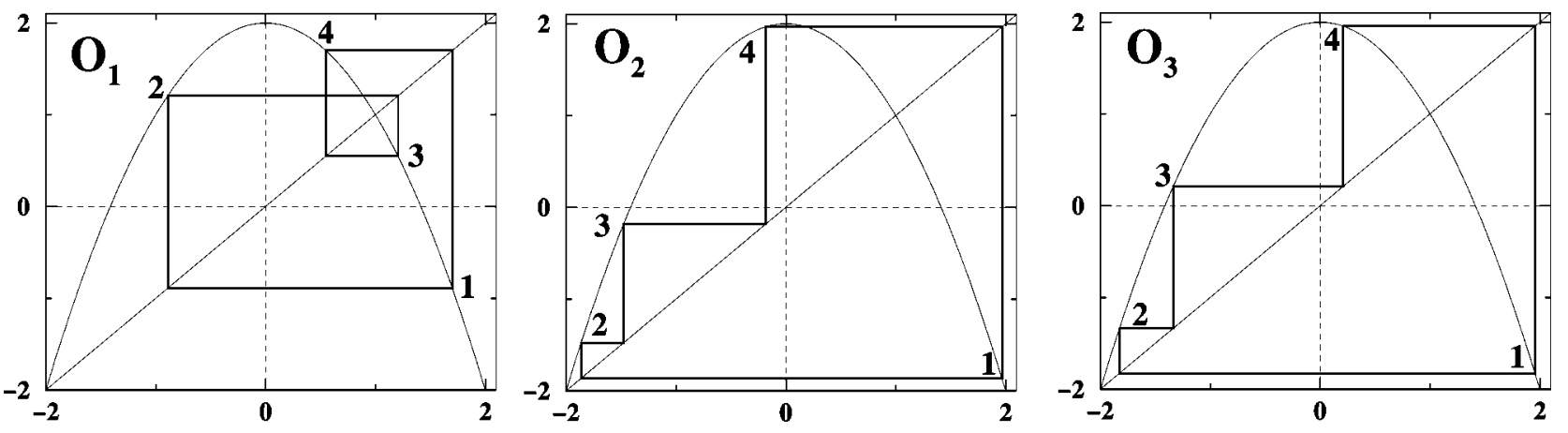

FIG. 1. Structure of the three orbits $O_{n}$, for $n=1,2,3$, when $a=2$ and $b=0$. Orbits $O_{2}$ and $O_{3}$ look quite similar, despite the strong dissimilarities of their analytical expressions, shown in Eqs. (19) and (20).

$$
\begin{gathered}
\sigma\left(\sigma^{2}-4 a+12\right)=0 \text { for } b=1, \\
\sigma\left(\sigma^{2}-4 a\right)=0 \text { for } b=-1 .
\end{gathered}
$$

These interesting equations show that the nondissipative (Hamiltonian) limit contains always an orbit for which the sum of orbital points is identically zero. The corresponding orbital equations simplify to

$$
\begin{gathered}
\left(x^{2}+2 x+a-2\right)\left(x^{2}-2 x+a-2\right)=0 \quad(b=1), \\
\left(x^{2}-a\right)^{2}=0 \quad(b=-1) .
\end{gathered}
$$

In the dissipative case, when $b \neq \pm 1$ (and $\sigma \neq 0$ ) the orbital equations may be obtained by eliminating $a$ among Eqs. (2) and (6). This procedure yields a fourth-degree polynomial in $x$, with coefficients that depend only on $b$ and $\sigma$ and whose roots give the following orbital points:

$$
\begin{aligned}
& x_{1}=\frac{1}{4}\left[\sigma-S_{1}-\sqrt{S_{2}\left(S_{1}+\sigma\right)}\right], \\
& x_{2}=\frac{1}{4}\left[\sigma+S_{1}-\sqrt{S_{2}\left(S_{1}-\sigma\right)}\right], \\
& x_{3}=\frac{1}{4}\left[\sigma-S_{1}+\sqrt{S_{2}\left(S_{1}+\sigma\right)}\right], \\
& x_{4}=\frac{1}{4}\left[\sigma+S_{1}+\sqrt{S_{2}\left(S_{1}-\sigma\right)}\right],
\end{aligned}
$$

where

$$
\begin{gathered}
S_{1} \equiv \sqrt{\sigma^{2}+4(1+b)^{2}}, \\
S_{2} \equiv 2\left(\frac{\sigma-2(1-b)}{\sigma}\right) S_{1} .
\end{gathered}
$$

Eliminating $\sigma$ between Eqs. (2) and (6) one obtains a 12th-degree polynomial in $x$ with coefficients depending only on $a$ and $b$. The three period- 4 orbits are formed by the roots of three fourth-degree polynomials that compose this 12th-degree polynomial.

In the remainder of the paper we explore Eqs. (2) and (6), our main analytical results.
For $(a, b)=(2,0)$, the well-studied limit in which the map is known to contain generating partitions, Eq. (6) reduces to $\left(\sigma^{2}-\sigma-4\right)(\sigma+1)=0$, giving us three roots, (1 $+\sqrt{17}) / 2,(1-\sqrt{17}) / 2$, and -1 which, substituted in Eq. (2), yield the orbits

$$
\begin{aligned}
O_{1}(x)= & x^{4}-\frac{1}{2}(1+\sqrt{17}) x^{3}-\frac{1}{2}(3-\sqrt{17}) x^{2} \\
& -(2-\sqrt{17}) x-1, \\
O_{2}(x)= & x^{4}-\frac{1}{2}(1-\sqrt{17}) x^{3}-\frac{1}{2}(3+\sqrt{17}) x^{2} \\
& -(2+\sqrt{17}) x-1, \\
O_{3}(x)= & x^{4}+x^{3}-4 x^{2}-4 x+1,
\end{aligned}
$$

respectively. Here and below, all square roots denote the positive value of the root. The orbital points for these three orbits are given in the Appendix.

Figure 1 shows the phase-space structure of the three orbits $O_{j}(x)$, from which one may read directly the corresponding symbolic codings.

Notice that, for any choice of parameters, just a single equation, Eq. (2), correctly parameterizes all possible period-4 orbits. In particular, Eq. (2) yields at once in factorized form the pair of conjugate orbits "interlaced" in the irreducible octic studied in Ref. [19],

$$
\begin{aligned}
O_{1}(x) O_{2}(x)= & x^{8}-x^{7}-7 x^{6}+6 x^{5}+15 x^{4}-10 x^{3}-10 x^{2} \\
& +4 x+1
\end{aligned}
$$

\section{B. General solution of the cubic}

For arbitrary parameter values, the solution of Eq. (6) depends essentially on a single number $N_{+}$:

$$
N_{+} \equiv \sqrt[3]{V+\sqrt{\Delta}}
$$

where $\Delta \equiv V^{2}-U^{3}$, a quantity that may also be written as

$$
\begin{aligned}
\Delta & =4(1-b)^{2}(1+b)^{4}-\left[\frac{4}{3} a-(1+b)^{2}\right]^{3} \\
& =\frac{16}{27} a^{2}\left[9(1+b)^{2}-4 a\right]-(1+b)^{4} \Delta_{1},
\end{aligned}
$$


TABLE I. Three possible choices of $\sigma_{k}$ in the generating partition limit, depending on the value chosen for the cubic root $N_{+}$. We fix $N_{+} \equiv N_{+}^{(1)}$.

\begin{tabular}{cccc}
\hline \hline$N_{+}$ & $\sigma_{1}$ & $\sigma_{2}$ & $\sigma_{3}$ \\
\hline$N_{+}^{(1)}$ & $\frac{1}{2}(1+\sqrt{17})$ & $\frac{1}{2}(1-\sqrt{17})$ & -1 \\
$N_{+}^{(2)}$ & $\frac{1}{2}(1-\sqrt{17})$ & -1 & $\frac{1}{2}(1+\sqrt{17})$ \\
$N_{+}^{(3)}$ & -1 & $\frac{1}{2}(1+\sqrt{17})$ & $\frac{1}{2}(1-\sqrt{17})$ \\
\hline \hline
\end{tabular}

$$
\Delta_{1}=4 a-\left(5 b^{2}-6 b+5\right)=4 a-4(1-b)^{2}-(1+b)^{2} .
$$

Fixing now the auxiliary quantities

$$
N_{-} \equiv U / N_{+},
$$

$\omega=-(1-i \sqrt{3}) / 2$, and $\omega^{2}=-(1+i \sqrt{3}) / 2$, one may write the three roots of Eq. (6) as

$$
\begin{gathered}
\sigma_{1}=N_{+}+N_{-}, \\
\sigma_{2}=\omega N_{+}+\frac{N_{-}}{\omega}=\omega N_{+}+\omega^{2} N_{-}, \\
\sigma_{3}=\omega^{2} N_{+}+\frac{N_{-}}{\omega^{2}}=\omega^{2} N_{+}+\omega N_{-} .
\end{gathered}
$$

In the generating partition limit $(a, b)=(2,0)$, Eq. (22) gives $N_{+}^{3}=(18+i \sqrt{51}) / 9$, allowing three possible choices for $N_{+}$, namely,

$$
\begin{gathered}
N_{+}^{(1)}=-\frac{1}{2}\left(1+\frac{i}{3} \sqrt{51}\right), \\
N_{+}^{(2)}=\omega N_{+}^{(1)} \\
N_{+}^{(3)}=\omega^{2} N_{+}^{(1)}
\end{gathered}
$$

These different choices simply show that there are three different possibilities for fixing the initial labelings of the $\sigma_{i}$ in Eqs. (26)-(28). The actual labelings underlying each possible choice are shown explicitly in Table I.

As it is not difficult to realize, the main wrinkles defining the stability of the system are those obtained when $\Delta=0$ and $\Delta_{1}=0$. Of interest also is the parameter locus along which $U=0$. All these loci are shown in Fig. 2

\section{The critical loci}

From Eq. (6) one recognizes that interesting changes in the dynamics occur for parameters such that $\Delta=V^{2}-U^{3}$ $=0$. Although this condition represents a simple curve in the $U \times V$ space, it implies rather different situations in the $a$ $\times b$ space. We consider them now.

(1) The locus $\Delta=0$ : As seen from Fig. $2, \Delta=0$ is the main wrinkle along which period-4 orbit is created, when $a$ increases. This locus has $N_{+}=N_{-} \equiv N=\sqrt[3]{V}$ $=\sqrt[3]{2(1-b)(1+b)^{2}}$ a fact that reduces Eq. (6) to $\sigma^{3}$

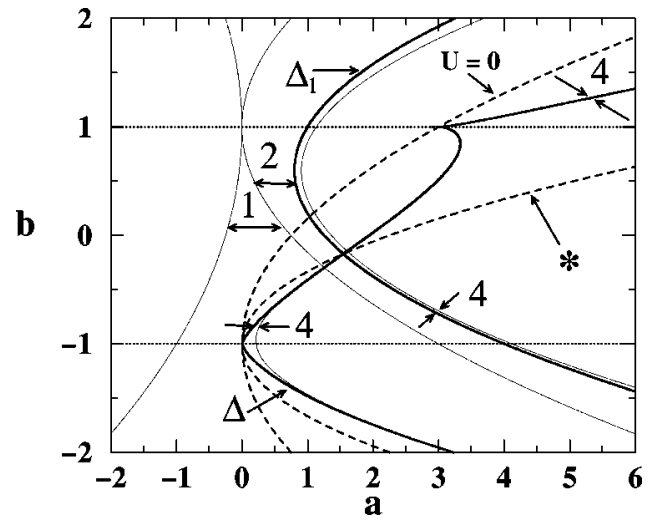

FIG. 2. Bifurcation curves $\Delta=0$ and $\Delta_{1}=0$, the wrinkles along which period- 4 orbit is created when $a$ increases. For reference, light curves indicate the location of well-known loci. The line indicated by an asterisk corresponds to the locus where $9(1+b)^{2}$ $-4 a=0$ [see Eq. (23)].

$-3 N^{2} \sigma-2 N^{3}=0$, with roots $\sigma=-N,-N, 2 N$. Along the locus $\Delta=0$ one finds the cuspidal structure shown in Fig. 3. The cusp is centered along the $b=1$ "Hamiltonian axis." In this figure, Greek letters represent interesting intersections points discussed in Ref. [21].

(2) The locus $\Delta_{1}=0$ : As seen in Fig. 2 , the $\Delta_{1}=0$ locus is the parabolic curve along which the period doubling $2 \rightarrow 4$ occurs. The intersection of $\Delta_{1}=0$ and $\Delta=0$ occurs at $(a, b)=(-9 \eta, \eta) \simeq(1.5441558,-0.1715728)$, where $\eta=$ $-3+\sqrt{2}$. This point is one of the vertices marking a domain where two different stable orbits of period four exist. For a discussion of this interesting domain see Ref. [21].

(3) The locus $U=0$ : As Eqs. (22) $-(25)$ show, $U=4 a / 3$ $-(1+b)^{2}=0$ implies either $N_{-}=0$ and $N_{+}=\sqrt[3]{[2 V}$ or $N_{-}=\sqrt[3]{[2 V}$ and $N_{+}=0$, depending on the sign of $V$, i.e., whether $b<1$ or $b>1$, respectively.

Two points along the curve $U=0$ are particularly noteworthy, namely $(a, b)=(3,1)$ and $(0,-1)$, points where Eq. (6) has a triply degenerate root $\sigma=0$. As shown in Fig. 3, at the first point there is cusp along the -1 eigenvalue locus. The curves $U=0$ and $\Delta_{1}=0$ intersect at $\left(6 b_{p}^{\prime}, b_{p}^{\prime}\right)$ and $\left(6 b_{u}^{\prime}, b_{u}^{\prime}\right)$, where $b_{p}^{\prime}=3-2 \sqrt{2} \simeq 0.171572$ and $b_{u}^{\prime}=3$

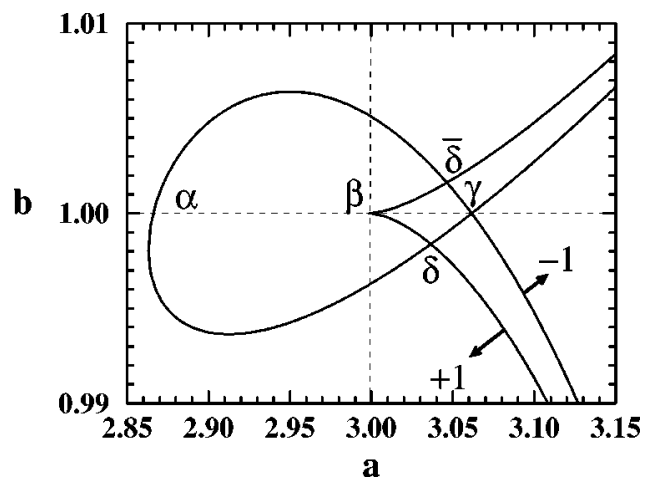

FIG. 3. Cuspidal structure along the "Hamiltonian axis" $b=1$. The numbers +1 and -1 refer to the eigenvalues characterizing the loci. 


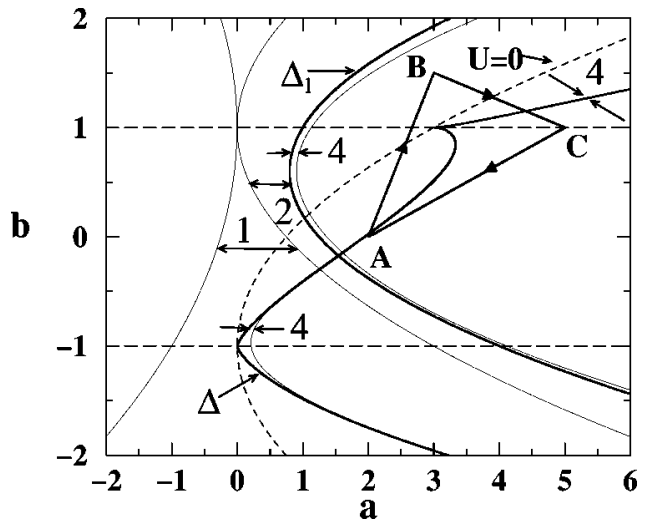

FIG. 4. Clockwise circulation along the triangle $A B C A$ enclosing the cuspidal structure shown in Fig. 3. The vertices are located at $(a, b)=(2,0),(3,3 / 2)$, and $(5,1)$. The dotted parabola $U=0$ is where discontinuous jumps occur when $b>1$ [see Eq. (45), below].

$+2 \sqrt{2} \simeq 5.828427$, both quantities being units $[22]$ in $Q(\sqrt{2})$ with norm +1 .

The curve indicated by an asterisk in Fig. 2 signals the location of the locus where $4 a-9(1+b)^{2}=0$ and, as Eq. (23) shows, signals the locus where $\Delta_{1}$ is the quantity ruling the square root that appears in $N_{+}$. There are two points where the asterisk curve meets the curve $\Delta_{1}=0$ : $\left(-9 b_{p}, b_{p}\right)$ and $\left(-9 b_{u}, b_{u}\right)$, where $b_{p}=-3+2 \sqrt{2} \simeq$ -0.171572 and $b_{u}=-3-2 \sqrt{2} \simeq-5.828427$, both numbers being also units in $\mathbb{Q}(\sqrt{2})$ with norm +1 . The point where $\Delta=0$ meets $\Delta_{1}$ coincides with the point where the curve indicated by an asterisk meets the curve $\Delta_{1}=0$. The perfect symmetry of all four intersections above reflects the remarkable property that all coordinates of intersections are functions of specific units in the field $Q(\sqrt{2})$.

We proceed now to investigate the changes observed while circulating along generic paths in parameter space.

\section{A CIRCULATION AROUND THE CUSP}

In this section we investigate orbital changes under a smooth clockwise circulation along the triangle $A B C A$ shown in Fig. 4. We start and return to the vertex $A=(2,0)$, the limit of complete binary trees and well-defined symbolic dynamics. The triangle $A B C A$ encloses the cuspidal structure shown in Fig. 3, which is triply degenerate, $\sigma^{3}=0$, for $(a, b)=(3,0)$. The three segments defining the triangle are $A B: \quad b=3 a / 2-3, B C: \quad b=-a / 4+9 / 4, C A: \quad b=a / 3$ $-2 / 3$. A similar adiabatic circulation in parameter space was considered earlier by Hansen [16] and Giovannini and Politi [17], who considered numerically two of the nine possible period-6 orbits of the Hénon map, showing the existence of discontinuities in the symbolic dynamics. Profiting from the analytical results obtained for the much simpler period-4 orbits, our main purpose here is (i) to characterize arithmetically the origin of such discontinuity as well as (ii) to locate in parameter space where exactly such discontinuity occurs.

At the vertex $A$, located at $(a, b)=(2,0)$, there are three real orbits. Moving upwards along $A B$ one arrives quickly at the point $a \simeq 2.024465632, b \simeq 0.03669844810$ where the segment $A B$ meets the locus $\Delta=0$. Arithmetically, this intersection is characterized by sextic numbers easily derived from the analytical expressions above. For $b$ $>0.03669844810$ there is one real and two complex orbits. This situation persists until the segment $B C$ crosses again the $\Delta=0$ locus, when all three orbits are real once again. Therefore, only one of the three period-4 orbits remains real during the whole clockwise circulation $A \rightarrow B \rightarrow C \rightarrow A$.

Figure 5 shows in detail the evolution of the real orbit during the circulation from $A \rightarrow B \rightarrow C \rightarrow A$. In this figure, numbers denote points of the orbit while dashed lines are used to help visualize how the points move in phase space. In Fig. 5(a) one sees clearly a crossing of the dashed lines, a crossing absent in Figs. 5(b)-5(d). In addition, comparing Figs. 5(a) and 5(d), which correspond to the vertex $A$ at the beginning and at the end of the circulation, one sees that the period-4 orbit changed continuously from $O_{1}(x)$ to $O_{2}(x)$, moving out of the generating partition parabola where complete binary tree exist and returning back to it but at a different location. Notice that the point 3 , which at the beginning of the circulation was between points 1 and 4 on the parabola, finished the circulation at a point lying between 4 and 2 on the parabola.

From Fig. 5 one recognizes an additional rather curious phenomenon happening along the path followed by the orbital point labeled by the number 3: this point crosses the diagonal in three different locations. Since the Hénon map is two dimensional, such crossings imply the existence of nontrivial orbits having $y_{i} \equiv x_{i}$. This means that the minimal polynomials defining orbital points of multidimensional systems may have multiple zeros, in sharp contrast with the usual Abelian equations [23], characteristic of onedimensional systems, which are not allowed to contain multiple roots [19]. Therefore, although by elimination of variables one may effectively reduce a multidimensional set of equations to an one-dimensional equivalent system, such reduced systems allow multiple roots a fact that may be used to segregate those one-dimensional equations of motion capable of representing higher-dimensional dynamical systems, a quite useful result.

The specific parameter values and orbital points of the three orbits having points $y_{i} \equiv x_{i}$ on the diagonal are summarized in Table II. Both orbits lying on the segment $A B$ have their parameter $a$ defined by a zero of the sextic

$$
\begin{gathered}
729 a^{6}-7776 a^{5}+34668 a^{4}-83952 a^{3}+117936 a^{2} \\
-91392 a+30208=0,
\end{gathered}
$$

while the remaining orbit lies on $B C$ and has its parameter $a$ defined by a zero of

$$
\begin{aligned}
& a^{6}-62 a^{5}+1607 a^{4}-23108 a^{3}+187775 a^{2}-773150 a \\
&+ 1184473=0 .
\end{aligned}
$$

Figure 6 shows the evolution of the four orbital points as a function of $a$ and, in particular, what happens in the vicinity of the three locations where the orbit contains points located on the diagonal $y_{i}=x_{i}$, i.e., when $x_{3}=x_{2}$. As it is clear, 

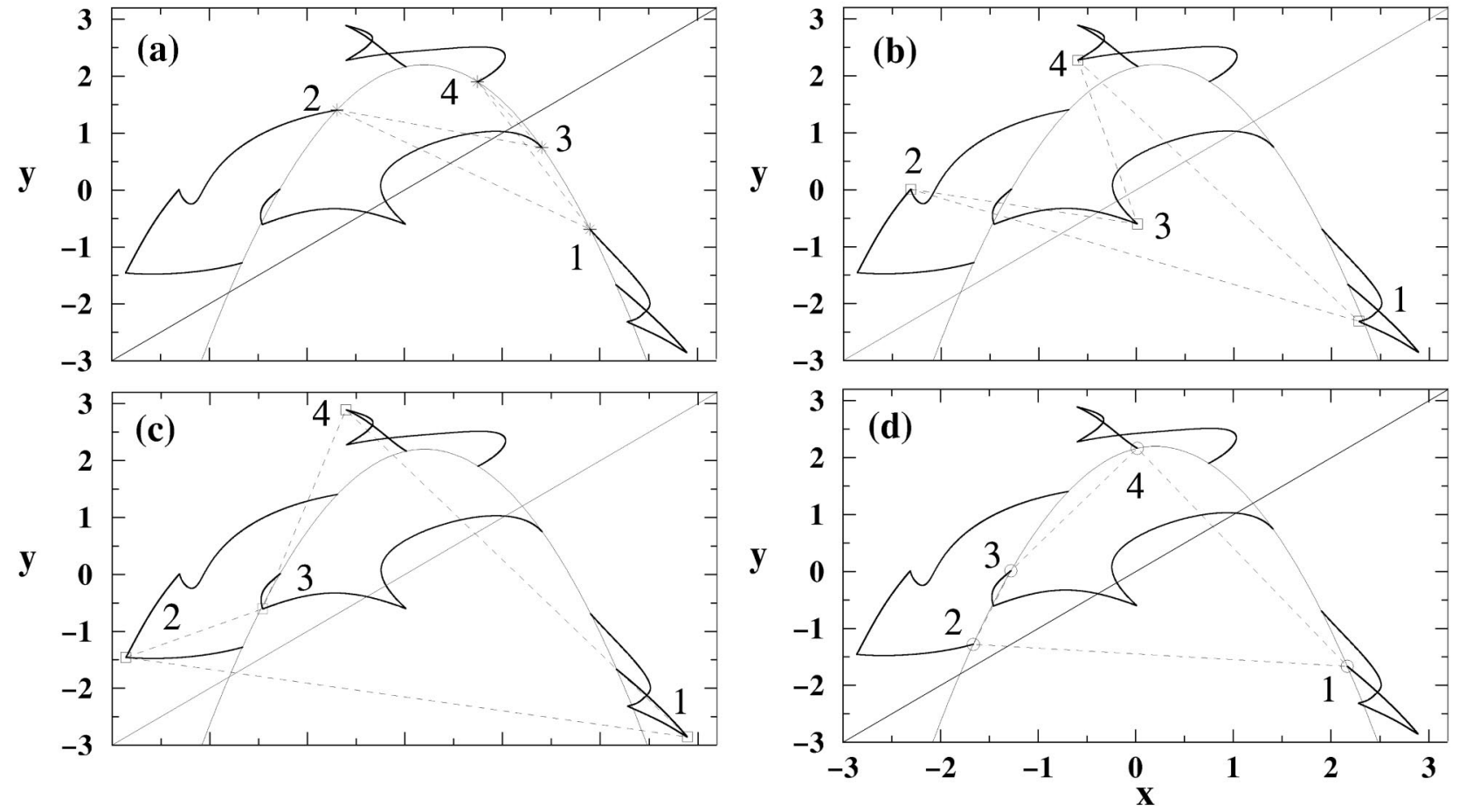

FIG. 5. Phase-space evolution, from $O_{1}(x)$ to $O_{2}(x)$, of the orbit that remains real during the clockwise circulation from $A \rightarrow B \rightarrow C$ $\rightarrow A$ along the triangle as shown in Fig. 4. Orbits start and finish on the logistic parabola. (a) The dashed line indicates the position of the initial orbit $O_{1}$ when departing from vertex $A$; (b) The orbit at vertex $B$; (c) The orbit at vertex $C$; (d) Arrival at $A$, with the final orbit $O_{2}$. The crossing of the dashed lines seen in (a) is topologically different from the situation in (b)-(d). Compare the orbital points $1,2,3,4$ with the corresponding ones in Fig. 1. The scales shown apply to all figure parts.

two intersections occur along the branch $A B$, one along $B C$, and none along $C A$. Recall that the branch $C A$ is fully contained in the domain where all three period- 4 orbits are real. As Fig. 6 shows, along this branch there is a relatively mild variation of the orbital points. In particular, one of them remains essentially constant from $a=5$ back to $a=2$, along $C A$.

Figure 6 shows clearly that as $a$ changes from $2 \rightarrow 5$ and back, the ordering of the orbital labels changes three times, the greatest variation happening in the segment $A B$, where two orbits are defined by complex numbers. The possibility of having "oscillations" in the symbolic dynamics seems not to have been noticed before. It would be nice to check whether or not there is any relation between such oscillations

TABLE II. Approximate numerical values of the three period-4 orbits shown in Fig. 7 having a point on the diagonal $y=x$. Exact values are roots of polynomials given in the text.

\begin{tabular}{lrrr}
\hline \hline & \multicolumn{1}{c}{$\mathcal{P}_{1}$} & \multicolumn{1}{c}{$\mathcal{P}_{2}$} & \multicolumn{1}{c}{$\mathcal{P}_{3}$} \\
\hline$a$ & 2.3470561315 & 2.8385632635 & 3.4785937435 \\
$b$ & 0.5205841972 & 1.2578448953 & 1.3803515641 \\
$\sigma$ & 2.1293267509 & -1.0450080961 & -1.5612688827 \\
$x_{1}$ & -1.6165475778 & -2.4464370758 & -2.6952633453 \\
$x_{2}$ & 0.8249554740 & -0.3935816003 & -0.5904586593 \\
$x_{3}$ & 0.8249554740 & -0.3935816003 & -0.5904586593 \\
$x_{4}$ & 2.0959633805 & 2.1885921804 & 2.3149117812 \\
\hline \hline
\end{tabular}

and the degree of the number fields underlying model parameters. This, however, seems to be a hard task.

Figure 7 shows the topological structure of the orbits that contain diagonal points $y_{i}=x_{i}$. Comparing Figs. 5(a) and 6(a) it is not difficult to realize that when $a$ increases from $A$ to $B$ along the segment $A B$, there is a very interesting situation in which three orbital points lie on a same straight line. The alignment of these three points marks the transition from "crossing" to "noncrossing" of the dashed lines, a significative change. The three-in-a-line alignment of the points 1,3 , and 4 happens for the parameters and orbit defined in the first column of Table III, which shows approximate values. The exact value of $b$ is a root of

$$
\begin{aligned}
p_{8}(b)= & 9 b^{8}+54 b^{7}+105 b^{6}-162 b^{5}-524 b^{4}-210 b^{3} \\
& +249 b^{2}-90 b+9
\end{aligned}
$$

derived from Eqs. (12), (14), and (15), from which one may obtain the other values exactly. Table III gives the location of another similar triple alignment, $b$ being now a root of

$$
p_{7}(b)=b^{7}+7 b^{6}-74 b^{4}-70 b^{3}+9 b-1 \text {. }
$$

Equations (34) and (35) have the symmetric group $S_{8}$ and $S_{7}$ and discriminants involving remarkably large primes for this context: $-2^{40} \times 3^{18} \times 223 \times 315496661$ and $2^{26} \times 8176981$, respectively. The signature [24] of $p_{8}(b)$ is $(2,3)$ while that of $p_{7}(b)$ is $(3,2)$. 

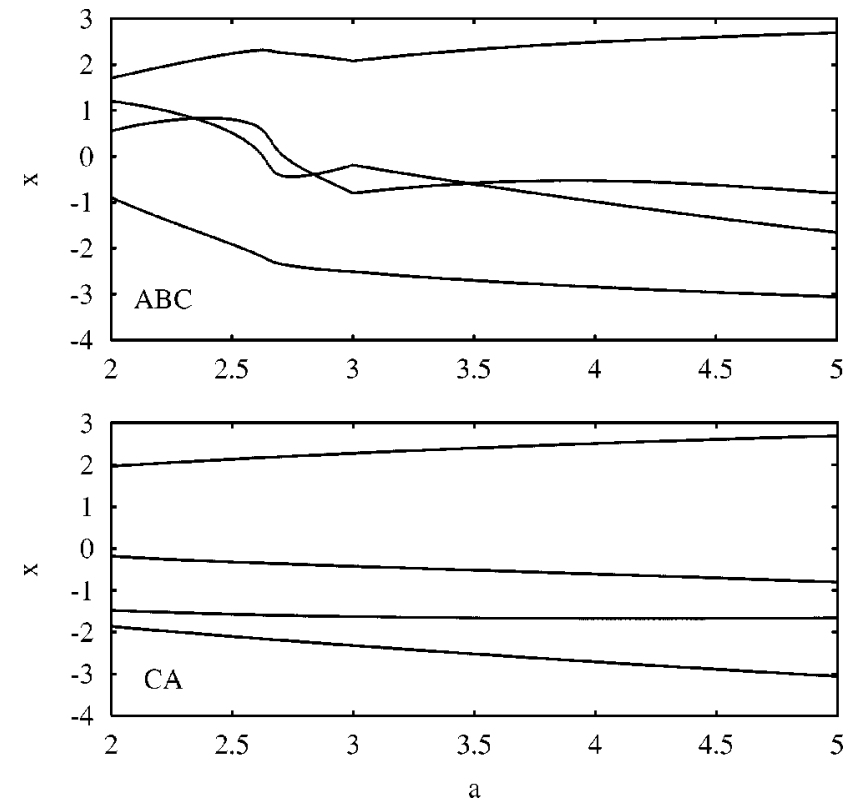

FIG. 6. Evolution of the four orbital points as function of $a$. The ordering of the labeling changes three times during the circulation. The location of the three crossings is given in Table II while the corresponding orbits are shown in Fig. 7.

For period six, the case discussed in Refs. [16,17], we find similar alignments at

$$
a=1.40268, \quad b=0.34800392,
$$

and

$$
a=1.2455525668, \quad b=0.3772237165 .
$$

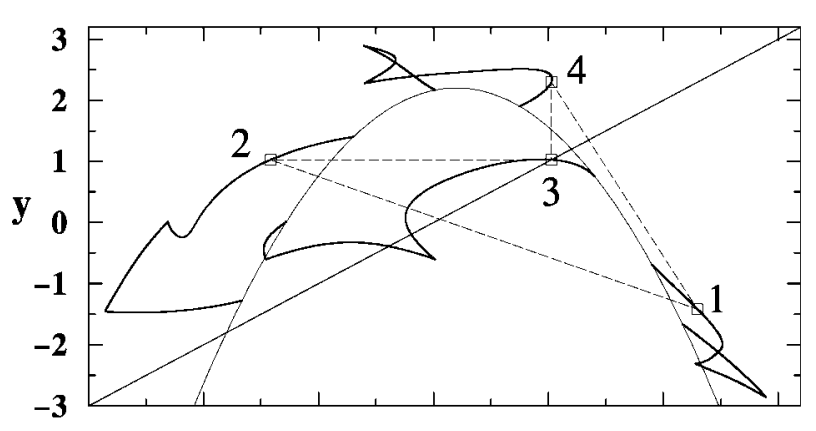

TABLE III. Approximate numerical values defining the two period-4 orbits characterized by three points lying on a straight line in phase space. Notice the occurrence of two $x$ values of the same magnitude. Exact values are roots of polynomials given in the text.

\begin{tabular}{lrr}
\hline \hline & Points 1,3,4 aligned & Points 2,3,4 aligned \\
\hline$a$ & 2.1021361633 & 2.6963243137 \\
$b$ & 0.1532042449 & 0.2321081045 \\
$\sigma$ & 2.4948791018 & -1.9638293467 \\
$x_{1}$ & 1.8240416733 & 2.1915688275 \\
$x_{2}$ & -1.1222167212 & -2.1915688275 \\
$x_{3}$ & 1.1222167212 & -1.5979687256 \\
$x_{4}$ & 0.6708374284 & -0.3658606210 \\
\hline \hline
\end{tabular}

Giovannini and Politi [17] find a homoclinic tangency for

$$
a=1.3569288, \quad[b=0.37298129],
$$

where brackets were used to indicate that $b$ (not given in Ref. [17]) was computed here so as to lie on the triangle. This tangency lies between those in Eqs. (36) and (37). However, when comparing the values above, recall that as shown by Newhouse [25], homoclinic tangencies occur in intervals in parameter space, not points, in sharp contrast with the arithmetical events discussed here that are intrinsically discrete.

\section{WHERE AND WHY DISCONTINUITIES OCCUR}

In this section, we show how the orbital discontinuity appears and compute the precise location on the triangle where such discontinuity happens. Although the argumentation here is for period-4 orbits, the same methodology ap-

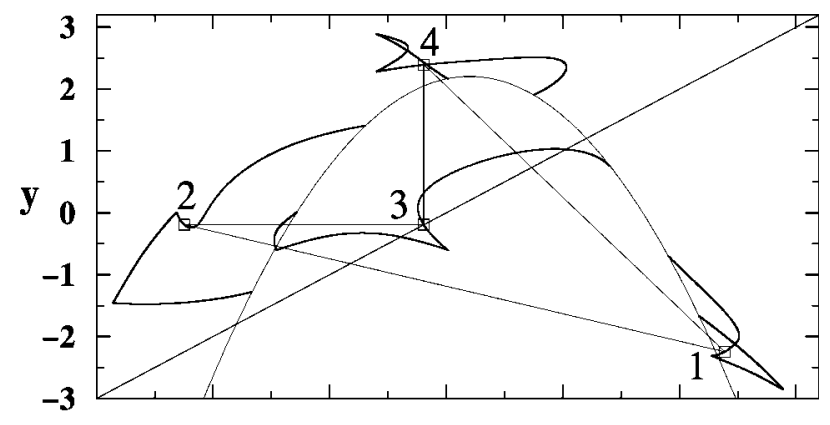

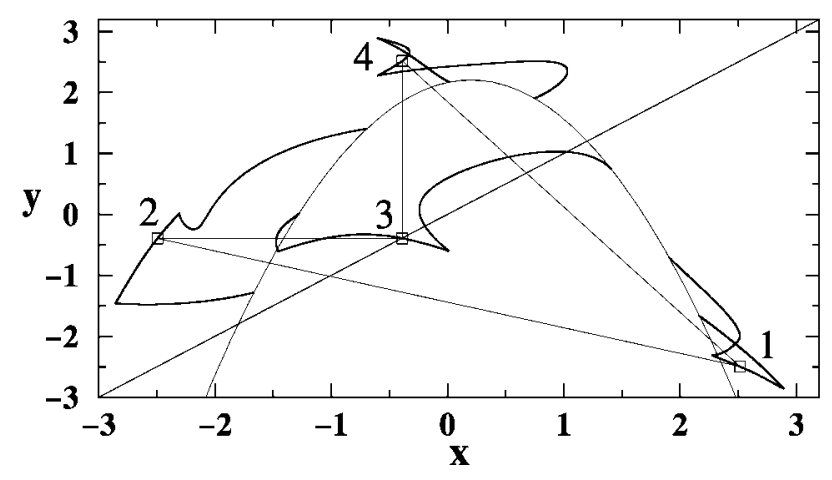

FIG. 7. Three orbits that have an orbital point on the diagonal $y=x$. They are all topologically equivalent since the polygons obtained by connecting successive orbital points with line segments do not exhibit the crossing that exists in Fig. 5(a). The scales shown apply to all figure parts. 
plies to orbits of any arbitrary periodicity.

To see how discontinuities appear, we start from the solutions of Eq. (22), writing them in polar coordinates

$$
N_{+}^{(n)}=r\left(\cos \frac{\theta}{3}+i \sin \frac{\theta}{3}\right) \omega^{n-1}, \quad n=1,2,3
$$

where

$$
r \equiv \sqrt[3]{|V+\sqrt{\Delta}|}
$$

and

$$
\begin{aligned}
& \cos \theta \equiv \operatorname{Re}(V+\sqrt{\Delta}) / r, \\
& \sin \theta \equiv \operatorname{Im}(V+\sqrt{\Delta}) / r,
\end{aligned}
$$

where $\operatorname{Re}(z)$ and $\operatorname{Im}(z)$ denote the real and imaginary parts of $z$, respectively. The corresponding new form of $\sigma_{n}$ is

$$
\begin{aligned}
\sigma_{n}= & N_{+}^{(n)}+U / N_{+}^{(n)}=r\left(\cos \frac{\theta}{3}+i \sin \frac{\theta}{3}\right) \omega^{n-1} \\
& +\frac{U}{r}\left(\cos \frac{\theta}{3}-i \sin \frac{\theta}{3}\right) \frac{1}{\omega^{n-1}} .
\end{aligned}
$$

When $\Delta>0$, Eqs. (40)-(41) simplify to

$$
\cos \theta=\frac{V+\sqrt{\Delta}}{|V+\sqrt{\Delta}|}=\operatorname{sgn}(V+\sqrt{\Delta}),
$$

$$
\sin \theta=0 .
$$

From Eq. (43) one sees that $\theta$ undergoes a discontinuous jump of $\pi$ whenever $V+\sqrt{\Delta}$ changes sign. The effect in $\sigma$ of this jump is to promote a permutation among the $\sigma$ values, implying a corresponding permutation of the orbits.

The aforementioned jump occurs for $V+\sqrt{\Delta}=V$ $+\sqrt{V^{2}-U^{3}}=0$, that is, for $U=0$ and $V+\sqrt{V^{2}}=V+|V|=0$ where $V=(1-b)(1+b)^{2}$. Therefore, the condition for the existence of a period-4 discontinuity is to have simultaneously

$$
U=0 \quad \text { and } \quad b>1
$$

Figure 8 shows the evolution of the three $\sigma_{n}$ values from $A \rightarrow B \rightarrow C$, where one can see where the discontinuity occurs. The jump occurs precisely at

$$
\begin{gathered}
a_{j}=\frac{1}{3}(71-8 \sqrt{55})=3.89013737, \\
b_{j}=\frac{1}{3}(-11+2 \sqrt{55})=1.277465658,
\end{gathered}
$$

when

$$
\sigma_{n}=-\frac{2}{3} w^{n-1} \sqrt[3]{-3748+508 \sqrt{55}}
$$
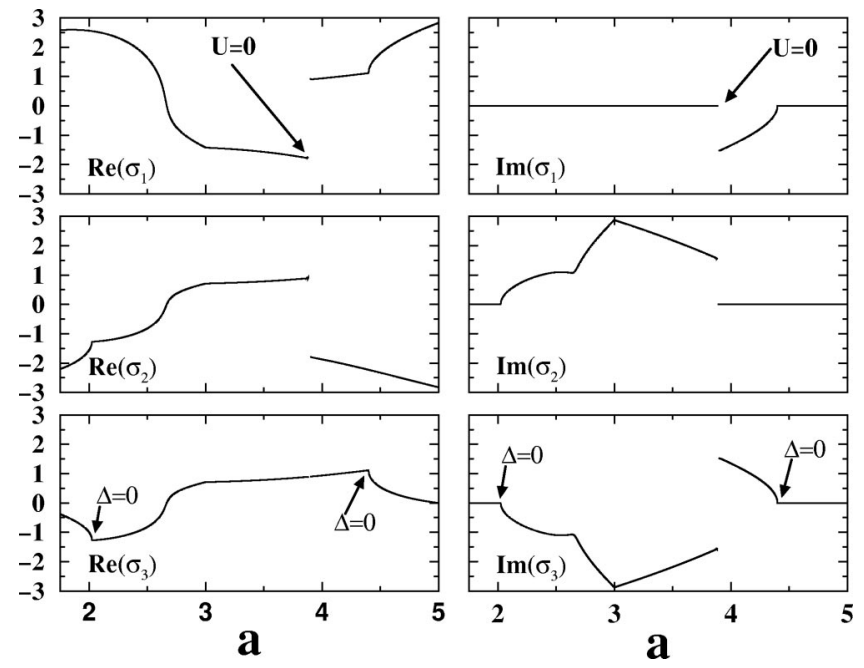

FIG. 8. The evolution of the three $\sigma$ values when moving from $A \rightarrow B \rightarrow C$ in the triangle in Fig. 4. The discontinuity occurs for the parameters $(a, b)$ given in Eqs. (46) $-(47)$, when $\left(\sigma_{1}, \sigma_{2}, \sigma_{3}\right)$ is permuted into $\left(\sigma_{2}, \sigma_{3}, \sigma_{1}\right)$.

The orbit corresponding to these parameters is shown in Fig. 9.

A significative point is that the discriminant $U=0$ of Eq. (45), although obtained in a rather different way, behaves precisely as the locus discussed by Giovannini and Politi [17] for period-6, in that both curves contain the cuspidal point.

When $\Delta<0$ all three values of $\sigma$ are real and there are no discontinuities.

\section{CONCLUSIONS}

This paper reported a fourth-degree polynomial capable of parametrizing simultaneously all three period-4 orbits of the Hénon map in terms of the sum $\sigma$ of orbital points, for arbitrary values of model parameters. The present analysis in phase space complements the corresponding parameter-space analysis done in Ref. [21] providing now a complete analytical description of all period-4 motions, valid for any real or complex orbit.

The parameterization described in this paper is very general and, in principle, may be done for orbits of any arbitrary periodicity of dynamical systems ruled by algebraic equa-

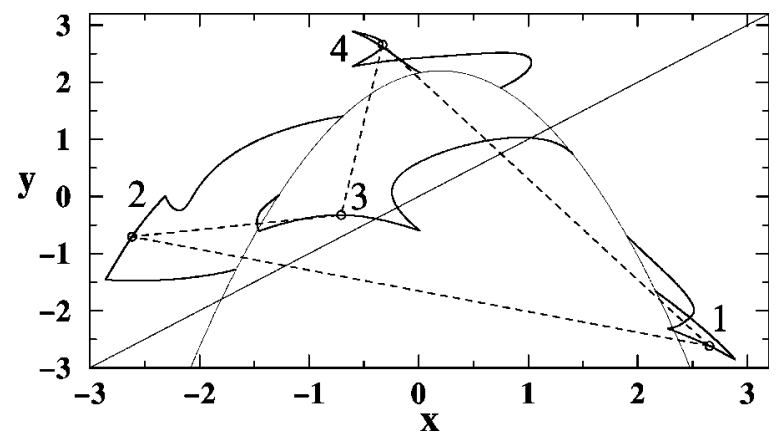

FIG. 9. Orbital structure at $\left(a_{j}, b_{j}\right)$, defined in Eqs. (46) and (47), where there is a discontinuous change of the $\sigma$ values. 
tions of motion. This means that the investigation of periodic motions, for arbitrary period $k$ and for any dynamical system ruled by algebraic equations of motion, may be effectively reduced to the investigation of just two polynomials: one defining all $k$-periodic orbits, similar to Eq. (2) above, and other defining the corresponding values of $\sigma$ for each orbit, similar to Eq. (6). In particular, we already computed [26] analytical expressions for the quintic and sextic polynomials that rule orbits of periods 5 and 6 .

From a theoretical point of view, an interesting and general result reported in this paper is the fact that minimal polynomials defining orbital points for multidimensional systems may have multiple zeros, in sharp contrast with the well-known Abelian equations [23] characteristic of onedimensional systems that are by no means allowed to contain multiple roots [19]. Therefore, one sees that the familiar Abelian equations underlying one-dimensional systems belong to the just the simplest class of equations of motion since there is a whole class of much more symmetrical equations still waiting to be properly characterized and classified.

Interesting analytical aspects of the orbits where considered in detail. In particular, we studied what happens with the three period- 4 orbits when they move adiabatically around continuous circuits in parameter space that includes a cuspidal structure. We find that when departing from wellknown generating partition limit $(a, b)=(2,0)$ with the orbit $O_{1}(x)$ and moving clockwise along the triangle shown in Fig. 4 one ends up on orbit $\mathrm{O}_{2}(x)$ upon arrival back at $(2,0)$. This phenomenon is similar to what was observed numerically for period-6 orbits by Hansen [16] who associated it with the existence of homoclinic tangencies. Such tangencies occur over intervals [25] and no exact analytical methods are known to locate them. In contrast, the analytical expressions obtained in this paper opened the possibility of characterizing arithmetically with precision (i) the parameter loci along which discontinuities occur as well as (ii) the origin of such orbital discontinuities that induce ambiguities in the symbolic coding.

Since discontinuities arise while circulating around cuspidal structures, it seems of interest to point out here a recent work of Carcassès and Kawakami [27] where the generic problem concerning the existence of a cusp point on a fold bifurcation curve was addressed for $n$-dimensional maps.

By adiabatically following trajectories we observed a phenomenon that is markedly different from what happens with the familiar Abelian equations that rule one-dimensional dynamical systems when compared with the more intrincate one-dimensional equations obtained by eliminating variables that represent higher-dimensional dynamics: higherdimensional systems may now contain one (or several) points located on the diagonal in phase space. For the twodimensional Hénon map under consideration this implies the existence of orbits containing points with $y_{i}=x_{i}$. Three examples are shown in Fig. 7.

Last but not least, as hinted by results in the Appendix, the great symmetry that interconnects the equations of conjugate orbits interconnects also physics and number theory in a novel and direct way. The analytic computation of trajectories automatically generates interesting number-theoretical results and equations for which general mathematical algorithms and theories are still lacking. In particular, since for even moderate periodicities the number fields underlying conjugate orbits already involve relatively high degrees, their investigation allows one to understand the intricate origins of many "miraculous" identities that exist in number fields of lower degrees and which are very hard to justify within the framework of the low fields where they live and where they appear to be just accidental events. Such identities are simply "echos" of symmetries living in much higher fields. In some sense, the arithmetical determination of orbital points may be regarded as a procedure inverse to that normally used in mathematics, where one moves from number fields of lowerdegree to higher-degree fields. Orbital symmetries become even more striking when the period increases [26].

\section{ACKNOWLEDGMENTS}

J.A.C.G. thanks Professor Hans-Jürgen Herrmann (Stuttgart and Paris), Professor Dietrich Wolf (Duisburg), and Professor Peter Grassberger (Jülich) for their kind interest and stimulating discussions. This work was supported by the bilateral project PROBRAL 0133/2001 sponsored by CAPES (Brazil) and DAAD (Germany). J.A.C.G. is a Senior Research Fellow of the CNPq, Brazil.

\section{APPENDIX: THE ARITHMETICAL STRUCTURE OF ORBITAL POINTS IN THE GENERATING PARTITION LIMIT}

This appendix collects exact analytical expressions for the orbital points of Eqs. (18)-(20) and summarizes their main arithmetical characteristics. As will become clear, the arithmetic structure of the orbital points and the number fields underlying the equations of motion raise a number of interesting questions not only in dynamics but also in number theory.

As before, square roots are to be taken as positive. As seen from Eq. (1), in the generating partition limit all three orbits obey $x_{i+1}=2-x_{i}^{2}$.

\section{The quartic orbit $O_{3}(x)$}

In this simpler case we have $\sigma=-1$ with the orbital points composing $\mathrm{O}_{3}(x)$, in Eq. (20), being

$$
\begin{aligned}
& x_{1}=\frac{1}{4}[-1-\sqrt{5}-\sqrt{30-6 \sqrt{5}}], \\
& x_{2}=\frac{1}{4}[-1+\sqrt{5}-\sqrt{30+6 \sqrt{5}}], \\
& x_{3}=\frac{1}{4}[-1-\sqrt{5}+\sqrt{30-6 \sqrt{5}}], \\
& x_{4}=\frac{1}{4}[-1+\sqrt{5}+\sqrt{30+6 \sqrt{5}}] .
\end{aligned}
$$


By introducing two convenient quantities, closely related to units in $Q(\sqrt{5})$,

$$
\xi \equiv \frac{1+\sqrt{5}}{4} \quad \text { and } \quad \eta \equiv \frac{1-\sqrt{5}}{4},
$$

the orbital points above may also be written as

$$
\begin{aligned}
& x_{1}=-\xi-\sqrt{3\left(1-\xi^{2}\right)}=-1.827090916, \\
& x_{2}=-\eta-\sqrt{3\left(1-\eta^{2}\right)}=-1.338261213, \\
& x_{3}=-\xi+\sqrt{3\left(1-\xi^{2}\right)}=0.2090569265, \\
& x_{4}=-\eta+\sqrt{3\left(1-\eta^{2}\right)}=1.956295202 .
\end{aligned}
$$

This remarkable symmetry exists due to automorphisms involving the outermost square roots

$$
\begin{gathered}
2 \xi \sqrt{1-\xi^{2}}=\sqrt{1-\eta^{2}}, \\
-2 \eta \sqrt{1-\eta^{2}}=\sqrt{1-\xi^{2}},
\end{gathered}
$$

which have the net effect of greatly simplifying the orbital dynamics.

\section{The conjugate octic orbits $O_{1}(x)$ and $O_{2}(x)$}

The decomposition into quadratic factors of both $O_{1}(x)$ and $O_{2}(x)$, in Eqs. (18) and (19), may be easily done by first decomposing $\sqrt{17}$, a quadratic number, into a product of two quartic factors, namely, $\sqrt{17}=\psi \varphi$, where

$$
\psi=\sqrt{17+4 \sqrt{17}}, \quad \varphi=\sqrt{17-4 \sqrt{17}} .
$$

Then, both orbits may be decomposed using either $\mathrm{Q}(\psi)$ or $\mathrm{Q}(\varphi)$.

After conveniently introducing an auxiliary unit $\alpha$,

$$
\alpha=\frac{\sigma_{1}}{2}=\frac{1}{4}(1+\sqrt{17}),
$$

closely connected with $\sigma_{1}$ defined in Table I, we obtain the following representation for the orbital points of $O_{1}(x)$ :

$$
\begin{aligned}
& r_{1}=\frac{1}{2}[\alpha+(1-\alpha) \psi]+\frac{1}{4} \sqrt{\beta+(\beta-4) \psi}, \\
& r_{2}=\frac{1}{2}[\alpha-(1-\alpha) \psi]+\frac{1}{4} \sqrt{\beta-(\beta-4) \psi}, \\
& r_{3}=\frac{1}{2}[\alpha+(1-\alpha) \psi]-\frac{1}{4} \sqrt{\beta+(\beta-4) \psi}, \\
& r_{4}=\frac{1}{2}[\alpha-(1-\alpha) \psi]-\frac{1}{4} \sqrt{\beta-(\beta-4) \psi},
\end{aligned}
$$

where $\beta=17-3 \sqrt{17}$. These points may be equivalently written in the form

$$
\begin{aligned}
& r_{1}=\frac{1}{2}[\alpha-(1+\alpha) \varphi]+\frac{1}{4} \sqrt{\beta+4 \alpha \varphi}, \\
& r_{2}=\frac{1}{2}[\alpha+(1+\alpha) \varphi]+\frac{1}{4} \sqrt{\beta-4 \alpha \varphi},
\end{aligned}
$$

$$
\begin{aligned}
& r_{3}=\frac{1}{2}[\alpha-(1+\alpha) \varphi]-\frac{1}{4} \sqrt{\beta+4 \alpha \varphi}, \\
& r_{4}=\frac{1}{2}[\alpha+(1+\alpha) \varphi]-\frac{1}{4} \sqrt{\beta-4 \alpha \varphi} .
\end{aligned}
$$

Analogously, for $O_{2}(x)$ we use the conjugate unit

$$
\bar{\alpha}=\frac{\sigma_{2}}{2}=\frac{1}{4}(1-\sqrt{17}),
$$

obtaining the orbital points

$$
\begin{aligned}
& \bar{r}_{1}=\frac{1}{2}[\bar{\alpha}+(1-\bar{\alpha}) \varphi]+\frac{1}{4} \sqrt{\bar{\beta}+(\bar{\beta}-4) \varphi}, \\
& \bar{r}_{2}=\frac{1}{2}[\bar{\alpha}-(1-\bar{\alpha}) \varphi]-\frac{1}{4} \sqrt{\bar{\beta}-(\bar{\beta}-4) \varphi}, \\
& \bar{r}_{3}=\frac{1}{2}[\bar{\alpha}+(1-\bar{\alpha}) \varphi]-\frac{1}{4} \sqrt{\bar{\beta}+(\bar{\beta}-4) \varphi} \\
& \bar{r}_{4}=\frac{1}{2}[\bar{\alpha}-(1-\bar{\alpha}) \varphi]+\frac{1}{4} \sqrt{\bar{\beta}-(\bar{\beta}-4) \varphi},
\end{aligned}
$$

where $\bar{\beta}=17+3 \sqrt{17}$ is the conjugate of $\beta$. Equivalently, the points above may be written as

$$
\begin{aligned}
& \bar{r}_{1}=\frac{1}{2}[\bar{\alpha}+(1+\bar{\alpha}) \psi]+\frac{1}{4} \sqrt{\bar{\beta}-4 \bar{\alpha} \psi}, \\
& \bar{r}_{2}=\frac{1}{2}[\bar{\alpha}-(1+\bar{\alpha}) \psi]-\frac{1}{4} \sqrt{\bar{\beta}+4 \bar{\alpha} \psi}, \\
& \bar{r}_{3}=\frac{1}{2}[\bar{\alpha}+(1+\bar{\alpha}) \psi]-\frac{1}{4} \sqrt{\bar{\beta}-4 \bar{\alpha} \psi}, \\
& \bar{r}_{4}=\frac{1}{2}[\bar{\alpha}-(1+\bar{\alpha}) \psi]+\frac{1}{4} \sqrt{\bar{\beta}+4 \bar{\alpha} \psi} .
\end{aligned}
$$

The symmetry enforced by the equations of motion depends critically on several unsuspected interconnections among octic numbers, which "propagate downwards," affecting quartic and quadratic fields when their members are suitably combined. For instance, in the quartic field containing

$$
\sqrt{17+4 \sqrt{17}} \text { and } \sqrt{17-4 \sqrt{17}}
$$

we find 


$$
\begin{aligned}
& (-3+\sqrt{17}) \sqrt{17+4 \sqrt{17}}=(5+\sqrt{17}) \sqrt{17-4 \sqrt{17}} \\
& (13-3 \sqrt{17}) \sqrt{17+4 \sqrt{17}}=(1+\sqrt{17}) \sqrt{17-4 \sqrt{17}}
\end{aligned}
$$

which imply, analogously to Eqs. (A10) and (A11),

$$
\begin{aligned}
& (-4+\sqrt{17}) \sqrt{17+4 \sqrt{17}}=\sqrt{17-4 \sqrt{17}}, \\
& (4+\sqrt{17}) \sqrt{17-4 \sqrt{17}}=\sqrt{17+4 \sqrt{17}},
\end{aligned}
$$

results that depend on the nonuniqueness of the decomposition in $Q(\sqrt{17}) \equiv Q(\psi \varphi)$ of the number

$$
\begin{aligned}
14-2 \sqrt{17} & =(1+\sqrt{17})(-3+\sqrt{17}) \\
& =(13-3 \sqrt{17})(5+\sqrt{17}) .
\end{aligned}
$$

Notice that, since

$$
\begin{gathered}
(-4+\sqrt{17})(1+\sqrt{17})=13-3 \sqrt{17} \\
(4+\sqrt{17})(-3+\sqrt{17})=5+\sqrt{17}
\end{gathered}
$$

Equation (A34) is obtained from Eq. (A33) by suitably multiplying it with $1 \equiv(-4+\sqrt{17})(4+\sqrt{17})$. In general, finding multiple decompositions implies having to deal with hard problems in Diophantine analysis [24,28]. What is particularly attractive here is that the octic dynamics underlying the equations of motion 'explains' tricky identities and nonuniqueness in quadratic number fields. In number theory, no systematic way of uncovering such identities is known yet [24].
[1] E. M. Bollt, T. Stanford, Y.-C. Lai, and K. Zyczkowski, Phys. Rev. Lett. 85, 3524 (2000); R. L. Davidchack, Y.-C. Lai, E. M. Bollt, and M. Dhamala, Phys. Rev. E 61, 1353 (2000).

[2] See the webbook written and maintained by the CATS cyclist team at http://www.nbi.dk/ChaosBook/

[3] J. Plumecoq and M. Lefranc, Physica D 144, 231 (2000).

[4] K. Zyczkowski and Y. C. Lai, Physica D 144, 197 (2000).

[5] Z. Zhou and S.-L. Peng, Physica D 144, 213 (2000).

[6] D. Sterling, H. R. Rubin, and J. D. Meiss, Physica D 134, 153 (1999).

[7] E. M. Bollt and M. Dolnik, Phys. Rev. E 55, 6404 (1997); Chaos 8, 702 (1998).

[8] L. Jaeger and H. Kantz, J. Phys. A 30, L567 (1997).

[9] K. T. Hansen and S. Güttler, J. Phys. A 30, 3421 (1997).

[10] F. Christiansen and A. Politi, Physica D 109, 32 (1997); Phys. Rev. E 51, R3811 (1995).

[11] P. Pollner and G. Vattay, Phys. Rev. Lett. 76, 4155 (1996).

[12] R. Badii, Chaos 7, 694 (1997).

[13] M. Eisele, J. Phys. A 32, 1533 (1999).

[14] P. Grassberger and H. Kantz, Phys. Lett. 113A, 235 (1985); P. Grassberger, H. Kantz, and U. Moenig, J. Phys. A 22, 5217 (1989).

[15] C. Mira, Chaotic Dynamics (World Scientific, Singapore, 1987), p. 351

[16] K. T. Hansen, Phys. Lett. A 165, 100 (1992).
[17] F. Giovannini and A. Politi, Phys. Lett. A 161, 332 (1992).

[18] J. A. C. Gallas, Europhys. Lett. 47, 649 (1999).

[19] J. A. C. Gallas, Phys. Rev. E 63, 016216 (2001).

[20] H. El Hamouly and C. Mira, C. R. Acad. Sci. (Paris) 293, 525 (1981); 294, 387 (1982); D. L. Hitzl and F. Zele, Physica D 14, 305 (1985); H. Yung-nien, Chin. Phys. Lett. 2, 97 (1985); Sci. Sin., Ser. A 29, 1302 (1986).

[21] A. Endler and J. A. C. Gallas, Physica A 295, 285 (2001).

[22] I. N. Stewart and D. O. Tall, Algebraic Number Theory, 2nd ed. (Chapman and Hall, London, 1994).

[23] N. H. Abel, J. Reine Angew. Math. 4, 131 (1829); G. B. Mathews, Algebraic Equations, 3rd ed., edited by W. E. H. Berwick (Cambridge University Press, Cambridge, 1930), Chap. 3; reprinted by Haffner, New York, 1960.

[24] H. Cohen, A Course in Computational Algebraic Number Theory, Graduate Texts in Mathematics, Vol. 138 (Springer, Berlin, 1993).

[25] S. P. Dawson, C. Grebogi, J. A. Yorke, I. Kan, and H. Koçak, Phys. Rev. E 48, 1676 (1993); Y.-C. Lai, C. Grebogi, J. A. Yorke, and I. Kan, Nonlinearity 6, 779 (1993); I. Kan, H. Koçak, and J. A. Yorke, Physica D 83, 313 (1995).

[26] A. Endler and J. A. C. Gallas (unpublished).

[27] J. P. Carcassès and H. Kawakami, Int. J. Bifurcation Chaos Appl. Sci. Eng. 9, 875 (1999), and references therein.

[28] A. Weil, Number Theory (Birkhäuser, Boston, 1984). 